\title{
Diabetes dietary management alters responses to food pictures in brain regions associated with motivation and emotion: a functional magnetic resonance imaging study
}

\author{
M. Chechlacz • P. Rotshtein • S. Klamer • K. Porubská • \\ S. Higgs • D. Booth • A. Fritsche - H. Preissl • H. Abele • \\ N. Birbaumer $\cdot$ A. Nouwen
}

Received: 27 June 2008 / Accepted: 25 November 2008 /Published online: 13 January 2009

(C) Springer-Verlag 2009

\begin{abstract}
Aims/hypothesis We hypothesised that living with type 2 diabetes would enhance responses to pictures of foods in brain regions known to be involved in learnt food sensory motivation and that these stronger activations would relate
\end{abstract}

Electronic supplementary material The online version of this article (doi:10.1007/s00125-008-1253-z) contains supplementary material, which is available to authorised users.

M. Chechlacz $\cdot$ P. Rotshtein $\cdot$ S. Higgs $\cdot$ D. Booth

A. Nouwen $(\triangle)$

School of Psychology, University of Birmingham,

Edgbaston,

Birmingham B15 2TT, UK

e-mail: a.nouwen@bham.ac.uk

S. Klamer $\cdot$ K. Porubská $\cdot$ H. Preissl $\cdot$ H. Abele MEG-Center, Eberhard-Karls-University of Tübingen,

Tübingen, Germany

A. Fritsche

Department of Internal Medicine,

Eberhard-Karls-University Tübingen,

Tübingen, Germany

H. Preissl

Department of Obstetrics and Gynecology,

University of Arkansas for Medical Sciences,

Little Rock, AR, USA

N. Birbaumer

Institute of Medical Psychology and Behavioral Neurobiology,

Eberhard-Karls-University of Tübingen,

Tübingen, Germany

N. Birbaumer

IRCCS Ospedale San Camillo,

Istituto di Ricovero e Cura a Carattere Scientifico,

Venezia Lido, Italy to scores for dietary adherence in diabetes and to measures of potential difficulties in adherence.

Methods We compared brain responses to food images of 11 people with type 2 diabetes and 12 healthy control participants, matched for age and weight, using functional magnetic resonance imaging (fMRI).

Results Having type 2 diabetes increased responses to pictured foods in the insula, orbitofrontal cortex (OFC) and basal ganglia and, within these regions, the effect of the fat content of the foods was larger in participants with type 2 diabetes than in healthy controls. Furthermore, increased activation to food within the insula and OFC positively correlated with external eating, dietary self-efficacy and dietary self-care. In contrast, responses within subcortical structures (amygdala and basal ganglia) were positively correlated with emotional eating and rated appetite for the food stimuli and negatively correlated with dietary self-care. Conclusions/interpretation Type 2 diabetes is associated with changes in brain responses to food that are modulated by dietary self-care. We propose that this is linked to the need to follow a life-long restrictive diet.

Keywords Amygdala - Basal ganglia - Dietary restraint .

Dietary self-care $\cdot \mathrm{fMRI} \cdot$ Food pictures $\cdot$ Insula .

Orbitofrontal cortex $\cdot$ Self-efficacy $\cdot$ Type 2 diabetes

$\begin{array}{ll}\text { Abbreviations } \\ \text { BOLD } & \text { Blood oxygen level-dependent } \\ \text { DEBQ } & \text { Dutch Eating Behaviour Questionnaire } \\ \text { DSCA } & \text { Dietary self-care activities } \\ \text { DSE } & \text { Dietary self-efficacy } \\ \text { fMRI } & \text { Functional magnetic resonance imaging } \\ \text { MNI } & \text { Montreal Neurological Institute } \\ \text { OFC } & \text { Orbitofrontal cortex }\end{array}$

Abbreviations

BOLD Blood oxygen level-dependent

DEBQ Dutch Eating Behaviour Questionnaire

DSCA Dietary self-care activities

DSE Dietary self-efficacy

fMRI Functional magnetic resonance imaging

OFC Orbitofrontal cortex 


\section{Introduction}

Dietary self-care is the cornerstone of self-management in people with type 2 diabetes. To reduce the risk of developing both short- and long-term physical complications, patients are encouraged to reduce their energy intake and to adopt a well-balanced, healthy diet that is low in fat and sugar and high in fibre. However, many individuals with diabetes have difficulty following this advice [1]. Moreover, those who do adhere to their dietary recommendations often report feeling deprived and having to deal with cravings for foods $[2,3]$. The disposition and incentive to eat not only increases during temporary deprivation of food, but chronic dietary restrictions in healthy people result in motivational, emotional and attitudinal changes that can lead to consumption of greater amounts of food and consequent weight gain $[2,4,5]$.

Neuroimaging studies have shown that food stimuli elicit responses in regions of the human brain that are associated with motivation and/or emotion, such as the orbitofrontal cortex (OFC) [6-9], insula [6-10], basal ganglia and amygdala [6, 7, 10-12]. In particular, sensory stimuli in foods that are rated as pleasant to eat or are high in energy elicit larger neural responses in these regions [6, 13-18].

However, these studies were carried out in healthy individuals. To our knowledge, there has been no investigation of the effects of living with type 2 diabetes on brain responses to food stimuli specifically in areas associated with motivation and emotion. Therefore, the main aim of the current study was to use functional magnetic resonance imaging (fMRI) to compare the brain responses to pictures of foods and visually matched pictures of non-foods of individuals with type 2 diabetes and group of healthy matched controls. Importantly, the food pictures varied in terms of fat and sugar content and size of portion (Fig. 1).

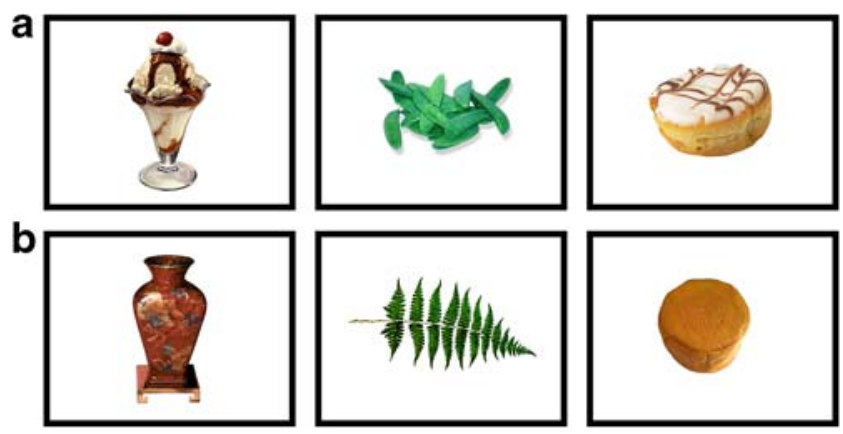

Fig. 1 Examples of visually matched food (a) and non-food (b) pictures used as stimuli in fMRI experiment and values for food measures used as covariates in data analysis. Portion sizes: ice cream $200 \mathrm{~g}(2.4 \mathrm{~g}$ fat, $13.2 \mathrm{~g}$ sugar); mangetout peas $100 \mathrm{~g}$ ( $0.2 \mathrm{~g}$ fat, $4.5 \mathrm{~g}$ sugar); iced cake $60 \mathrm{~g}$ (19.8 g fat, $30.7 \mathrm{~g}$ sugar)
In addition, as only limited information exists on the factors that influence on the ability of individuals with type 2 diabetes to adhere to their dietary recommendations, we examined whether brain responses to food stimuli were related to measures of dietary self-care [19] and potential difficulties in adherence, in particular, dietary self-efficacy [20] and dietary restraint [21].

Self-efficacy is the belief in one's ability to follow given courses of action - in this case, following dietary recommendations - and has been shown to predict dietary selfcare behaviour [20]. Dietary restraint is the conscious effort to limit food intake [21]. Restrained eaters who lack effective strategies are liable to give up their efforts when faced with a challenge [4]. Such lapses could arise from susceptibility to temptations to eat (external eating [4, 22]) or from habits of using food in attempts to reduce distress (emotional eating [23]).

We hypothesised that, compared with the non-diabetic controls, the group with type 2 diabetes would show larger neural responses to the pictures of foods in brain regions known to be involved in learnt food sensory motivation and in diet-related emotionality, and that these stronger activations would be related to scores for dietary self-care in diabetes and to measures of potential difficulties in dietary self-care, such as external and emotional eating habits.

\section{Methods}

\section{Participants}

Twelve participants with type 2 diabetes mellitus (one lefthanded) and 12 age- and BMI-matched healthy controls without diabetes (one left-handed; see Table 1 for details) were recruited through the diabetes clinic of the Endocrinology Department of the University Hospital Tübingen. None of the participants had a history of any neurological or psychiatric disorders. Selection criteria for participants with type 2 diabetes included (1) being diagnosed with type 2 diabetes for at least 3 years, (2) having experienced no major changes in diabetes-related medication for the past 6 months, and (3) having no other major health problems. One participant with diabetes had an almost complete agenesis of the corpus callosum, and the data collected for this participant were excluded from the analysis.

To avoid the occurrence of peak postprandial hyperglycaemia in individuals while in the scanner, they were asked not to eat for at least $3 \mathrm{~h}$ before the scheduled scanning. They were fully informed about the nature of the study and signed a written consent form before participation. The study was approved by the Medical Ethics Committee of the University of Tübingen. 
Table 1 Participant characteristics and behavioural scores

\begin{tabular}{|c|c|c|}
\hline Characteristic & $\begin{array}{l}\text { Type } 2 \\
\text { diabetes }^{\mathrm{a}}\end{array}$ & Controls ${ }^{\mathrm{b}}$ \\
\hline Age (years) & $55.36 \pm 14.94$ & $46.58 \pm 15.58$ \\
\hline BMI $\left(\mathrm{kg} / \mathrm{m}^{2}\right)$ & $32.86 \pm 4.94$ & $30.38 \pm 4.20$ \\
\hline Blood glucose $(\mathrm{mmol} / \mathrm{l})$ & $7.70 \pm 2.2^{\mathrm{c}}$ & $5.45 \pm 0.86$ \\
\hline $\mathrm{HbA}_{1 \mathrm{c}}(\%)$ & $7.59 \pm 1.65$ & NA \\
\hline Duration of diabetes (years) & $6.3 \pm 4.3$ & NA \\
\hline Diabetes treatment $(n)$ & & NA \\
\hline Tablets alone & 5 & \\
\hline Insulin & 2 & \\
\hline Insulin + tablets & 4 & \\
\hline \multicolumn{3}{|l|}{ Time since last meal (h) } \\
\hline Mean & $2.7 \pm 0.9$ & $4.3 \pm 3.3$ \\
\hline Median & 2.5 & 3.0 \\
\hline $\begin{array}{l}\text { Current disposition to eat } \\
\text { (VAS, } 0-100 \mathrm{~mm})^{\mathrm{e}}\end{array}$ & $5.55 \pm 4.93$ & $7.24 \pm 8.68$ \\
\hline \multicolumn{3}{|l|}{ DEBQ } \\
\hline Emotional eating & $0.88 \pm 0.50$ & $1.14 \pm 1.05$ \\
\hline External eating & $1.77 \pm 0.41$ & $2.00 \pm 0.78$ \\
\hline Restrained eating & $2.01 \pm 0.58$ & $1.91 \pm 0.59$ \\
\hline DSE & $55.2 \pm 25.2$ & NA \\
\hline DSCA ( $z$ scores $)$ & $-0.13 \pm 0.60$ & NA \\
\hline \multicolumn{3}{|l|}{ Food picture ratings } \\
\hline Desire to eat/appetite & $2.60 \pm 0.74^{\mathrm{d}}$ & $3.22 \pm 0.52$ \\
\hline Valence & $2.99 \pm 0.63$ & $3.27 \pm 0.52$ \\
\hline Arousal & $2.48 \pm 0.58$ & $2.91 \pm 0.57$ \\
\hline \multicolumn{3}{|l|}{ Non-food picture ratings } \\
\hline Valence & $2.34 \pm 0.66$ & $2.23 \pm 0.60$ \\
\hline Arousal & $1.94 \pm 0.68$ & $1.93 \pm 0.54$ \\
\hline
\end{tabular}

Values are means \pm SD

NA, not applicable; VAS, visual analogue scale

${ }^{a} n=11$ : seven men, four women

${ }^{\mathrm{b}} n=12$ : nine men, three women

${ }^{\mathrm{c}} p=0.008$ vs controls

${ }^{\mathrm{d}} p=0.03$ vs controls

${ }^{\mathrm{e}}$ Current disposition to eat is rated as hunger

Stimuli, task and procedure

Physical and psychological measures Prior to fMRI scanning, blood glucose levels were measured by the finger prick method using an Accu-Check Aviva (Roche Diagnostics, Mannheim, Germany). The weight (kg) and height $(\mathrm{cm})$ of each participant were measured wearing clothes but without shoes to calculate BMI. Each participant's current disposition to eat food was assessed by rating hunger on a $10 \mathrm{~cm}$ visual analogue scale from not at all (scored 0 ) to very much (scored 10) and participants indicated time since last meal. $\mathrm{HbA}_{1 \mathrm{c}}$ and treatment modality were obtained from the patients' hospital records (Table 1).

Patterns of emotional eating (desire to eat when in an emotional state), external eating (how tempted one is by food) and dietary restraint (how restrained one feels about eating) were assessed using the Dutch Eating Behaviour Questionnaire (DEBQ) [21]. Participants with diabetes were assessed for dietary self-efficacy (DSE) [20] and for dietary self-care activities (DSCA; dietary subscale of the Summary of Diabetes Self-Care Activities questionnaire) over the 7 days preceding the study [19].

Following scanning, participants rated the pictures of foods and non-foods (described below) for valence and arousal on a 1 (low) to 5 (high) Self-Assessment Manikin scale [24]. The food pictures were also rated for desire to eat (appetite) on a 5-point Likert scale ranging from 1 (not appetising) to 5 (very appetising).

Differences between the two groups of participants concerning age, BMI, blood glucose, hunger ratings, DEBQ scores and post-scanning ratings (appetite, valence and arousal) were calculated using a two-tailed, independent samples Student's $t$ test, assuming unequal variance. We used Matlab 7.2 (The MathWorks, Natick, MA, USA) and SPSS15 (SPSS, Chicago, IL, USA) for statistical analysis.

\section{fMRI experiment}

Stimuli A total of 36 various food pictures and 36 non-food visually matched (in terms of shape, complexity, brightness and colour) control pictures were used (Fig. 1). The nonfood control pictures were images of objects not related to food, such as plants, a car, pillow, ball and furniture. The food pictures included images of fries, fried chicken, cakes, salads and fresh fruit. The healthy diet recommended for individuals with type 2 diabetes is based on decreased sugar, fat and overall food intake. Therefore, to test whether these factors modulate brain responses, the foods depicted in the pictures varied in their content of fat and sugar and in volume (portion size); these variations were quantified using German food composition tables and validated by a dietitian from the Diabetes Clinic at the University Hospital Tübingen.

Experimental procedure During scanning, the food and non-food stimuli were presented in a pseudo-random order using an event-related design. Each stimulus was presented for $2 \mathrm{~s}$ followed by an inter-stimulus interval of between $6 \mathrm{~s}$ and $12 \mathrm{~s}$. Each stimulus was presented once. The experiment was split into three scanning sessions. Participants were instructed to look carefully at each presented picture during the scanning.

fMRI data acquisition A Siemens 1.5 T scanner (Siemens Vision, Erlangen, Germany) equipped with a standard headcoil was used to acquire blood oxygen level-dependent (BOLD) contrast-weighted echo-planar images during the functional scans. Thirty nine axial slices (4 mm thick with a 
$1 \mathrm{~mm}$ gap) were acquired with a $64 \times 64$ pixel matrix and an in-plane resolution of $3 \times 3 \times 5 \mathrm{~mm}^{3}$, a $90^{\circ}$ flip-angle, an echo time (TE) of $40 \mathrm{~ms}$ and a repetition time (TR) of $2 \mathrm{~s}$. After the functional scans, a high-resolution T1-weighted structural image $(1 \times 1 \times 1 \mathrm{~mm}$ resolution) was acquired for co-registration and display of the functional data. Although the inter-participant time of scanning was variable, there was no between-group difference $(p>0.1)$; time of testing for the diabetes group ranged from 10:45 till 19:15 hours, and for the controls ranged from 09:45 till 19:05 hours.

Analysis of fMRI data Echo-planar image volumes were spatially realigned and unwrapped to correct for movement artefacts, transformed to the Montreal Neurological Institute (MNI) standard space using an advanced procedure to normalise and segment [25], and smoothed using a $9 \mathrm{~mm}$ Gaussian kernel to account for residual inter-participant differences using SPM5 (Wellcome Department of Imaging Neuroscience, London, UK; www.fil.ion.ucl.ac.uk/spm, released 1 December 2005). Finally, to improve the signalto-noise ratio an independent component analysis (ICA) was performed using Melodic FSL (FMRIB, Oxford, UK) to identify artefacts that could not be explained by movement variables with a frequency that was outside the stimuli frequency range (2-8 Hz [26]). For each session one ICA component was selected and used as a regressor of no interest in the subsequent analyses.

A whole-brain voxel-based analysis was performed with SPM5. For each participant we used a general linear model to estimate the response for each condition. The experimental design matrix included a regressor depicting the onset of each condition (food, non-food) and covariates for the individual rating of arousal and valence for each picture. For the food condition we also included covariates that describe the fat content, sugar content, volume and individual appetite ratings for each stimulus. All these regressors were convolved with the canonical haemodynamic response function, [27] and regressors modelling their time derivatives were also included. Regressors of no interest included the movement variables, the ICA component and harmonics that capture low-frequency changes in the signal (i.e. high pass filtering with frequency cut-off of $1 / 128 \mathrm{~Hz}$ ).

For each participant we computed contrast images representing the effect of stimuli (food $>$ non-food), main effect of volume, fat and sugar content, and of appetite ratings. To generalise to the population level, the resulting contrasts were used in a second-level whole brain analysis with two sample Student's $t$ test (type 2 diabetes vs control participants), with participants as random variables. Note that to control for the possibility of diabetes-related vascular deterioration, which could significantly affect the estimated BOLD responses, and for non-specific effects of medication in participants with diabetes [28], between- group comparisons were made only using interactions (i.e. using images that reflect the differences between conditions within each participant, e.g. food $>$ non-food; effect of the amount of fat in the food pictures). Finally, we also tested for correlations between brain responses that were modulated by self-reported adherence to dietary recommendations (DSCA), self-efficacy (DSE), DEBQ scores, time since last meal and current disposition to eat, rated as hunger. For the latter measure we used BMI and blood glucose levels as covariates. Unequal variance and independent samples were assumed in these models.

In accordance with previous work on food-related stimuli, our regions of interest were the OFC, insula, basal ganglia (putamen, caudate, nucleus accumbens) and amygdala. Brain regions are based on the Duvernoy Human Brain Atlas [29]. For simplicity we did not differentiate substructures within our regions of interests. We report and interpret results based on the gross anatomical definition of these regions. We used a combined peak and cluster size threshold [30] with a peak significance of $z>3(p<0.0017)$ at a cluster size larger than $45 \mathrm{~mm}^{3}$ at $p<0.005$. Effects outside our regions of interest and above our threshold are reported in the Electronic supplementary material (ESM).

\section{Results}

Eating pattern traits, current disposition to eat and appetite for pictured foods

Healthy controls and participants with diabetes did not differ significantly in scores on the DEBQ scales of emotional, external or restrained eating; or current disposition to eat, rated as hunger; or time since last meal ( $p>0.1$ in all cases).

Ratings for the visual stimuli after scanning differed between pictures of food and non-food. Positive valence and arousal were both greater for food than for non-food (valence, $t[d f 42.5]=-4.9, p<0.0001$; arousal, $t[d f 42.8]=$ $-4.8, p<0.0001$ ), but there were no significant differences between the two groups.

In contrast, eating-specific ratings of food pictures showed a group effect. Participants with type 2 diabetes rated food pictures as less appetizing $(t[d f 17.7]=-2.3$, $p<0.05$ ) (Table 1), with no difference in SD between groups (both 1.2 \pm 0.3 ) (Table 1).

Blood glucose and BMI

Participants with diabetes had significantly higher levels of blood glucose than did controls who were matched in age and BMI $(t[12.7]=-3.15, p<0.01)$ (Table 1$)$. This degree of 
hyperglycaemia has been found not to affect the BOLD contrast [31]. Blood glucose level was not correlated with any of the above psychological measurements (all $p$ values $>0.1$ ) or with the neural response to food $>$ non-food. BMI did not significantly differ between groups and was not correlated with any of the above behaviour measurements $(p>0.1)$; it also did not modulate brain responses to food (>no-food) within our regions of interest.

\section{Activation of brain regions}

Across all participants, larger responses for food stimuli than for non-food stimuli were observed in the right insula/ operculum, left OFC (and at a lower threshold of significance [LTS] in the right OFC (MNI $x y z$ coordinates: [33 $54-15], z=2.62$ ) and right basal ganglia (BG; Table 2, Fig. 2; ESM Table 1) observed also on the left BG nuclei at lower threshold of statistical significance (MNI [ $\left.\begin{array}{lll}-6 & 12 & -3\end{array}\right]$, $z=1.93$ ). Interestingly, the food-specific responses within each of these regions were significantly greater in participants with type 2 diabetes than in controls (Table 2, Fig. 2;
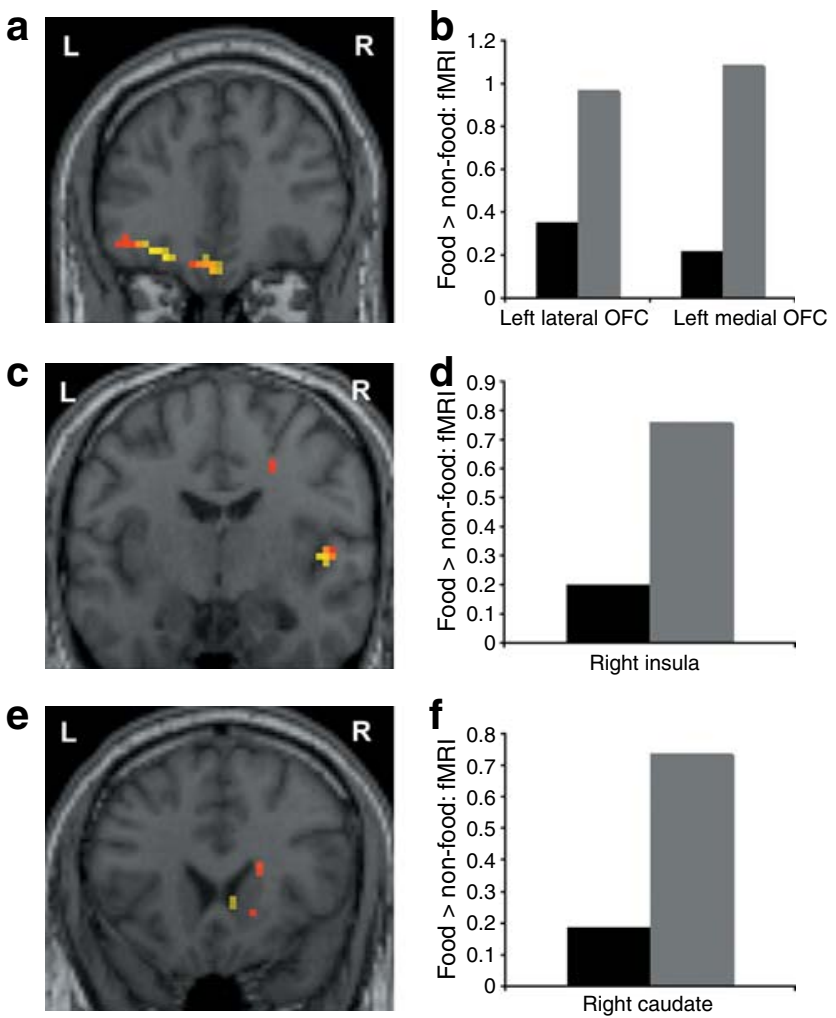

Fig. 2 Coronal view of statistical parametric mappings showing food $>$ non-food within the OFC (a), insula (c) and basal ganglia (e). Yellow clusters show significant increases in responses for food $>$ non-food across groups; red clusters show larger responses in participants with diabetes compared with controls for food $>$ non-food. The plots $(\mathbf{b}, \mathbf{d}, \mathbf{f})$ show the effect size of the difference between food $>$ non-food BOLD response for each region in controls (black bars) and participants with type 2 diabetes (grey bars)
ESM Table 1). Exclusion of the two left-handed participants (one control and one participant with diabetes) did not alter these results (ESM Fig. 1a-c), which concurs with the lack of significant lateralisation in brain responses when the threshold was lowered. Therefore, the results presented include all participants regardless of handedness.

The fat content of the pictured foods had a larger effect on brain responses in participants with type 2 diabetes than in controls. This was observed in the left medial OFC extended bilaterally, left insula/operculum (and in the right, LTS [45 - 15 15], $z=1.9$ ), the left caudate (and in the right, LTS [24 -27 9], $z=2.17$ ) (Table 2) - regions partly overlapping with those showing the effect for food generally. Taking the two groups together, fat content had no significant effect above threshold.

In contrast, across the groups, increased levels of sugar in the pictured foods correlated with increased responses in the right medial OFC and right caudate (Table 2), but the groups did not differ reliably in modulation by sugar content.

The volume of food did not modulate responses within our regions of interest, either across or between the groups, although volume greatly affected responses in visual cortex in both groups (ESM Table 2).

Across all participants, rated appetite for each food in the pictures correlated positively with responses to the food (>non-food) stimuli within the basal ganglia (putamen and nucleus accumbens) (Table 2). This effect was larger for participants with diabetes than for controls in the caudate bilaterally (Table 2). In contrast, time since last meal did not modulate neural responses to food $>$ no-food in our prespecified regions of interest.

Relationships of brain activation with dietary management

To examine the role of food motivation in general and in the dietary management of diabetes, we correlated the foodspecific responses in the regions of interest with the psychological measures. Again, for completeness we report the results of a whole brain voxel-based analysis in the ESM (ESM Tables 3-6).

Responses to the food stimuli in the OFC, insula and left amygdala correlated positively across the two groups with ratings for current disposition to eat. This effect was most pronounced in the bilateral insula (left: MNI [ $-42-12$ 24], $z=3.21$, cluster $=48 \mathrm{~mm}^{3}$; right: MNI $[42-15-6], z=2.96$, cluster $=60 \mathrm{~mm}^{3}$; left OFC: MNI [ $\left.\begin{array}{lll}-15 & 36 & -21\end{array}\right], z=3.91$, cluster $>900 \mathrm{~mm}^{3}$ ); left amygdala: MNI $\left[\begin{array}{lll}-30 & -3 & -24\end{array}\right]$, $z=3.37$, cluster $=228 \mathrm{~mm}^{3}$ ). Furthermore, responses of the bilateral medial OFC (MNI [ $\left.\begin{array}{lll}-9 & 60 & -15\end{array}\right], z=3.89$, cluster $=360 \mathrm{~mm}^{3}$ ) (Fig. 3a, b) correlated with external eating, whereas activation by food stimuli in the right caudate (MNI $\left[\begin{array}{lll}18 & 0 & 9\end{array}\right], z=3.4$, cluster $=417 \mathrm{~mm}^{3}$ ) correlated with emotional/comfort eating (Fig. 3c, d). 


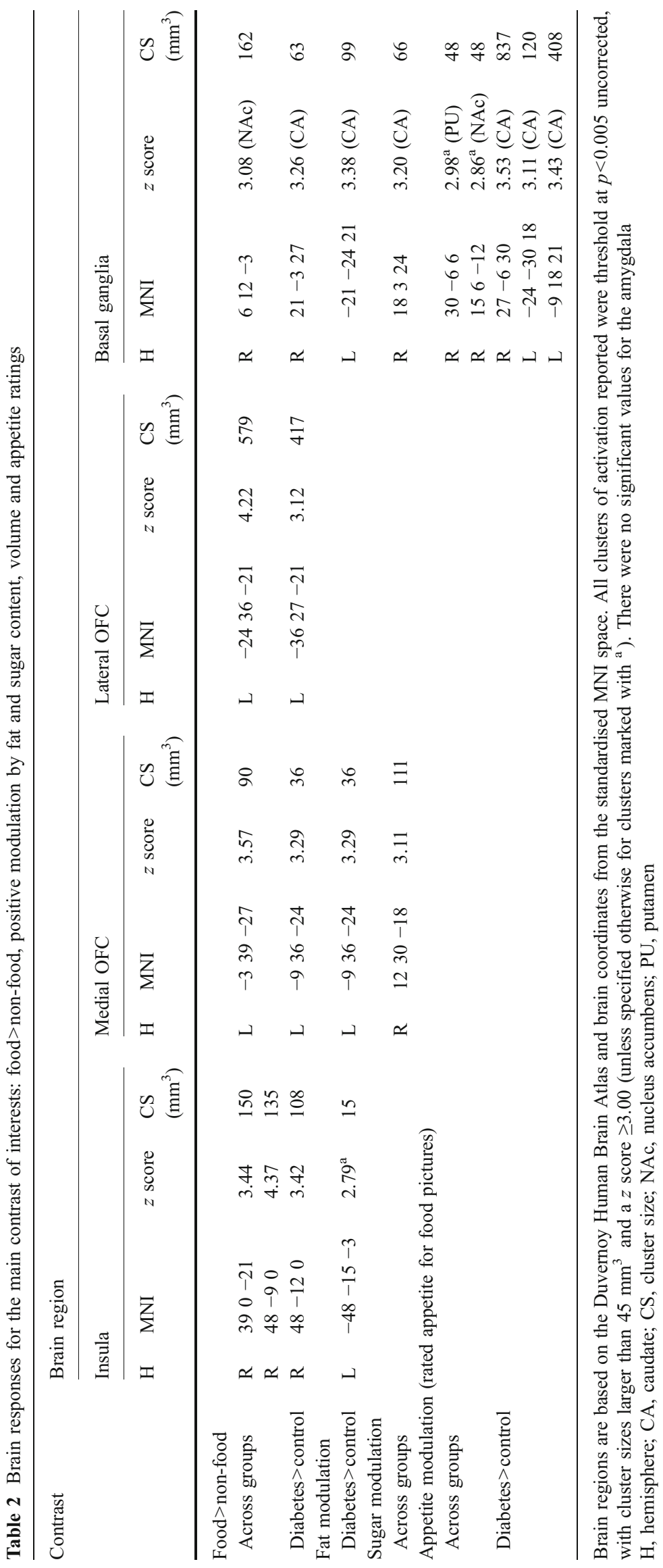



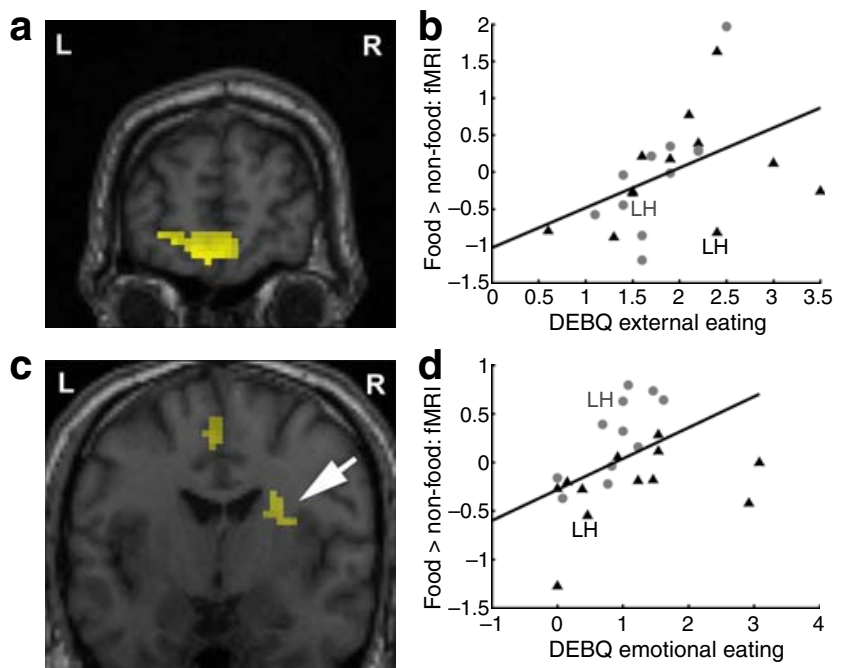

Fig. 3 Coronal view of statistical parametric mappings depicting significant correlations across groups between brain responses to food $>$ non-food and external eating behaviour within the OFC $(r=0.45)$ (a) and emotional eating behaviour in the basal ganglia $(r=0.49)(\mathbf{c})$. b, d Brain responses (food $>$ non-food) of the aforementioned regions plotted against the behavioural indices in controls (black triangles) and participants with type 2 diabetes (grey circles). LH, left-handed participants

Finally, in participants with type 2 diabetes, foodspecific responses in the right OFC $(r=0.6)$ and left insula $(r=0.8)$ were positively correlated with DSE, i.e. confidence in ability to follow the dietary recommendations for type 2 diabetes (Table 3, ESM Fig. 2a, b). In addition, responses in the right lateral OFC were positively correlated with scores for DSCA ( $r=0.69)$ (Table 3, Fig. 2c), whereas the right amygdala $(r=-0.79)$, left putamen $(r=-0.83)$ and left nucleus accumbens $(r=-0.78)$ each showed a negative correlation with dietary self-care (Table 3, ESM Fig. 2d, e).

\section{Discussion}

Our data indicate that people with type 2 diabetes show stronger activation to food pictures in the insula, OFC and basal ganglia than do non-diabetic controls. In addition, within these regions we found that the fat content of food stimuli had a larger effect on responses in participants with type 2 diabetes. An important finding of this study is that this increased activation to food within the insula and OFC correlated positively with dietary adherence, dietary selfefficacy and with external eating. In contrast, activation in subcortical structures, including the amygdala, caudate, putamen and nucleus accumbens, was positively correlated with emotional eating scores and rated appetite for the pictured foods, and negatively correlated with dietary adherence. Such results indicate that type 2 diabetes is associated with changes in brain responses to food stimuli. These changes are associated with dietary self-care activities.
Involvement of the insula and $\mathrm{OFC}$ and basal ganglia in responses to food pictures reported in this study and the correlation of this activity with rated current disposition to eat is consistent with previous reports. The insula is a primary gustatory area $[32,33]$ and is activated by the thought of tasting food, as evoked by deprivation of food [9, 18, 34, 35]. Similarly, the OFC is well known as a secondary taste area and is involved in the learnt integration of visual, gustatory and olfactory inputs [32, 33, 36, 37] and shows a decline of activation with eating (sensory-specific satiety). Single-cell recording studies show that neurons in the OFC (and in the amygdala) respond to the sight, taste and smell of food. This food-related neuronal activity varied with hunger and its satiation, implicating these cells in motivational processing of stimuli in foods [38-41].

Numerous neuroimaging studies indicate that activation of regions within the basal ganglia (putamen, caudate and nucleus accumbens) is associated with food pleasantness and hunger-induced changes in desirability of foods [7, 12, 42, 43]. Previous studies measuring brain responses in healthy participants have shown increases in responses in the insula, basal ganglia and OFC that are greater to highenergy food than to low-energy food $[6,44,45]$. This is in accordance with our finding that the sugar content of the pictured foods modulated responses in the basal ganglia and medial OFC.

The novelty of the present study lies in the analysis of relationships between food-specific activation and psychological processes relevant to healthy eating by participants with type 2 diabetes. Activation in the insula and OFC by the sight of food was positively correlated with external eating, predisposition to eat, dietary self-efficacy and dietary adherence. That is to say, these regions showed a larger response to food in individuals who were more tempted by food yet were also more confident of their ability to deal successfully with dietary restrictions, and hence adhered better to the recommended diet. The simplest interpretation of this pattern is that the strong response to food in the OFC and insula is associated with successful resistance to temptations to eat and, as a result, better dietary adherence. This is in line with the proposal that the medial OFC restrains immediate desires in favour of longterm outcomes [46-48]. In contrast, food-specific activation in subcortical structures, including the amygdala (greater in diabetes), caudate, putamen and nucleus accumbens, was negatively correlated with dietary adherence. The activation in these structures correlated positively with rated appetite for the pictured foods and scores for emotional eating. A strong desire for food, especially emotional overeating, would be expected to disrupt adherence to dietary recommendations, and so the obvious conclusion is that the amygdala and basal ganglia are critical to such difficulties in dietary self-management. 


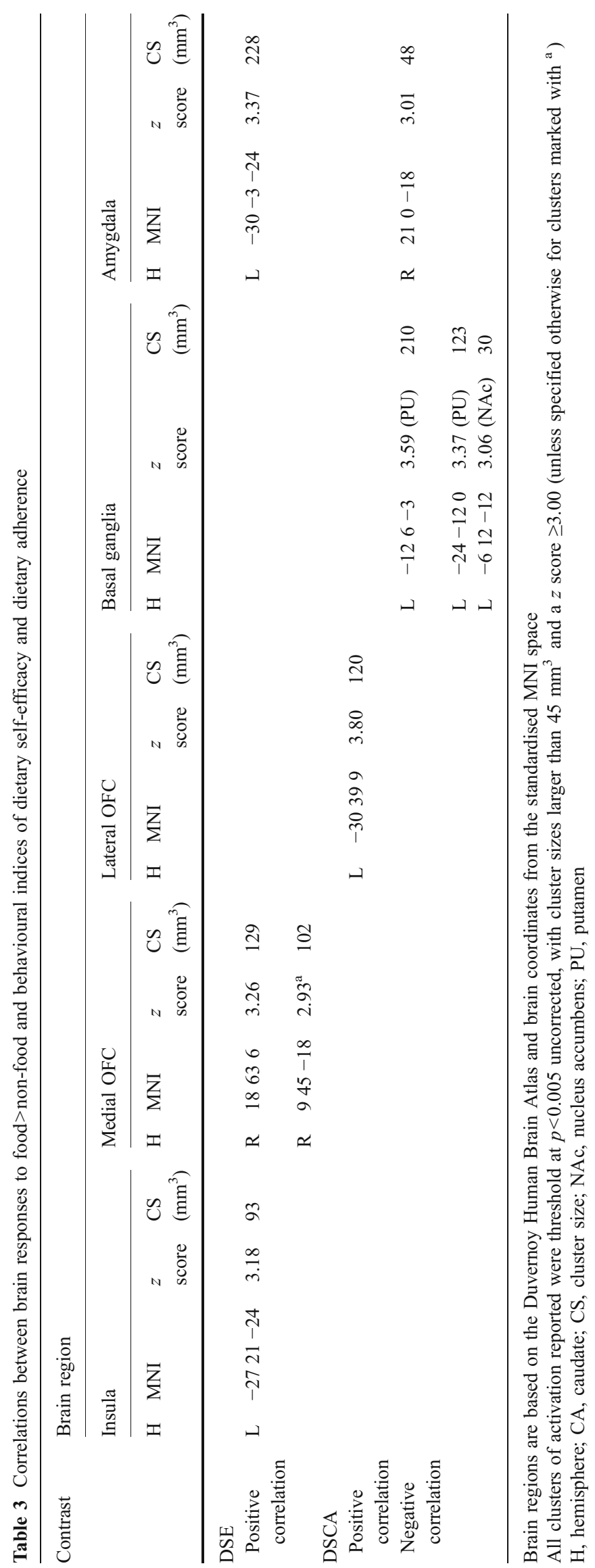


One line of interpretation is that successful dietary adherence, boosting dietary self-efficacy, depends in part on inhibiting emotionally based eating responses to thoughts of the sensory attractions of foods, particularly items that reduce the healthiness of the diet because they are high in fat or sugar. Thus, successful diet adherence may depend on the OFC inhibiting responses to food in subcortical structures, a role that is consistent with reports of OFC functions (see $[49,50]$ ). Support for such potential inhibitory processes in diabetes comes from the finding that the ratings for appetite for food were lower in participants with type 2 diabetes than in the healthy group, whereas BOLD responses to food were found to be greater in those with type 2 diabetes in many regions of the brain. This is readily explained by appetite for food being not limited to the sensory and somatic motivation reflected in those activations. Some of the social motivation for eating those foods, especially for refusing them, is not likely to be reflected by those brain responses. These findings may also point to differences between explicit and implicit food preferences in individuals with type 2 diabetes.

This study is subject to a number of shortcomings. The mean time period since the last meal was greater in the control group than in the diabetes group. The difference was not statistically significant and there was no difference in hunger rating between the groups. However, the possibility remains that these time differences may have influenced the results and may partly explain why the participants in the control group rated the food images as more appetising than did those in the diabetes group. Despite lower scores in appetising ratings in the diabetes group, brain activations to food stimuli in regions associated with sensory motivation were stronger in the diabetes group than in the control group. Together with lack of modulation of brain activations by time since the last meal, it indicates that the differences in time since last meal had little or no effect on the greater activations to food stimuli in regions associated with sensory motivation in the diabetes group than in the control group.

It is also important to note that our participants with diabetes had less than adequate control of their diabetes. Although blood glucose levels were not significantly related to any of the ratings or BOLD responses within the regions of interest, it is possible that higher blood glucose levels may have affected the results. Furthermore, we note that previous literature has only indicated effects of reduced BOLD responses following hypoglycaemia [51], while one study showed that acute hyperglycaemia has no significant effect on the BOLD response [29]. To our knowledge, no studies performed to date have examined how chronic hyperglycaemia affects the BOLD response and, specifically, whether it affects the neural response to food stimuli. Further research is needed to examine these possible effects.
Future research should also examine how medication affects the BOLD response and whether it influences neural responses to food stimuli in particular. In the present study, participants with diabetes used a variety of agents to control their diabetes and we cannot rule out the possibility that these agents affected the specific activations of the brain that we observed.

In summary, activation in response to food pictures in brain regions known to be involved in motivation and in emotional processing are stronger in participants with type 2 diabetes than in healthy controls. These regions also mediate adherence to dietary recommendations and dietary selfefficacy in diabetes, in particular, the struggle to stick to a healthy diet, especially by reducing fat intake. The observed pattern of activation relates in specific ways to the motivational, emotional and attitudinal changes known to be associated with chronic dietary restrictions [4, 5]. Further work on regional activation and connectivity, coupled with psychological investigation of the particular affective processes involved, is likely to improve understanding of difficulties in dietary adherence in type 2 diabetes. We suggest that similar alterations in motivation and emotion involved in eating could occur in other chronic disorders that require long-term dietary adjustments, such as cardiac disease and obesity, and could therefore be barriers to successful management of the disease.

Acknowledgements This work was partially supported by the Federal Ministry of Education and Research (BMBF 01GI0837) and by the German Science Foundation (DFG KFO-114).

\section{References}

1. Ary DV, Toobert D, Wilson W, Glasgow RE (1986) Patient perspective on factors contributing to nonadherence to diabetes regimen. Diabetes Care 9:168-172

2. Hall RF, Joseph DH, Schwartz-Barcott D (2003) Overcoming obstacles to behavior change in diabetes self-management. Diabetes Educ 29:303-311

3. Yannakoulia M (2006) Eating behavior among type 2 diabetic patients: a poorly recognized aspect in a poorly controlled disease. Rev Diabet Stud 3:11-16

4. Polivy J, Herman CP (1985) Dieting and binging. A causalanalysis. Am Psychol 40:193-201

5. Raynor HA, Epstein LH (2003) The relative-reinforcing value of food under differing levels of food deprivation and restriction. Appetite 40:15-24

6. Killgore WDS, Young AD, Femia LA, Bogorodzki P, Rogowska J, Yurgelun-Todd DA (2003) Cortical and limbic activation during viewing of high- versus low-calorie foods. Neuroimage 19:13811394

7. Porubská K, Veit R, Preissl H, Fritsche A, Birbaumer N (2006) Subjective feeling of appetite modulates brain activity: an fMRI study. Neuroimage 32:1273-1280

8. Simmons WK, Martin A, Barsalou LW (2005) Pictures of appetizing foods activate gustatory cortices for taste and reward. Cereb Cortex 15:1602-1608 
9. Wang GJ, Volkow ND, Telang F et al (2004) Exposure to appetitive food stimuli markedly activates the human brain. Neuroimage 21:1790-1797

10. Hinton EC, Parkinson JA, Holland AJ, Arana FS, Roberts AC, Owen AM (2004) Neural contributions to the motivational control of appetite in humans. Eur J Neurosci 20:1411-1418

11. Morris JS, Dolan RJ (2001) Involvement of human amygdala and orbitofrontal cortex in hunger-enhanced memory for food stimuli. J Neurosci 21:5304-5310

12. Pelchat ML, Johnson A, Chan R, Valdez J, Ragland JD (2004) Images of desire: food-craving activation during fMRI. Neuroimage 23:1486-1493

13. Beaver JD, Lawrence AD, van Ditzhuijzen J, Davis MH, Woods A, Calder AJ (2006) Individual differences in reward drive predict neural responses to images of food. J Neurosci 26:5160-5166

14. Gottfried JA, O'Doherty J, Dolan RJ (2003) Encoding predictive reward value in human amygdala and orbitofrontal cortex. Science 301:1104-1107

15. Kringelbach ML, O'Doherty J, Rolls ET, Andrews C (2003) Activation of the human orbitofrontal cortex to a liquid food stimulus is correlated with its subjective pleasantness. Cereb Cortex 13:1064-1071

16. O'Doherty JP, Deichmann R, Critchley HD, Dolan RJ (2002) Neural responses during anticipation of a primary taste reward. Neuron 33:815-826

17. Rolls ET, McCabe C (2007) Enhanced affective brain representations of chocolate in cravers vs. non-cravers. Eur J Neurosci 26:1067-1076

18. Small DM, Zatorre RJ, Dagher A, Evans AC, Jones-Gotman M (2001) Changes in brain activity related to eating chocolate: from pleasure to aversion. Brain 124:1720-1733

19. Toobert DJ, Glasgow RE (1994) Assessing diabetes self-management: the summary of diabetes self-care activities. In: Bradley C (ed) Handbook of psychology and diabetes: a guide to psychological measurement in diabetes research and practice. Harwood, London, pp 351-375

20. Senécal C, Nouwen A, White D (2000) Motivation and dietary self-care in adults with diabetes: are self-efficacy and autonomous self-regulation complementary or competing constructs. Health Psychol 19:452-457

21. Van Strien T, Frijters JER, Bergers GPA, Defares PB (1986) The Dutch Eating Behavior Questionnaire (DEBQ) for assessment of restrained, emotional, and external eating behavior. Int $\mathrm{J}$ Eat Disord 5:295-315

22. Van Strien T, Van de Laar FA (2008) Intake of energy is best predicted by overeating tendency and consumption of fat is best predicted by dietary restraint: a 4-year follow-up of patients with newly diagnosed type 2 diabetes. Appetite 50:544-547

23. Blair AJ, Lewis VJ, Booth DA (1990) Does emotional eating interfere with success in attempts at weight control. Appetite 15:151-157

24. Bradley MM, Lang PJ (1994) Measuring emotion: the selfassessment manikin and the semantic differential. J Behav Ther Exp Psychiatry 25:49-59

25. Ashburner J, Friston KJ (2003) Image segmentation. In: Frackowiak RSJ, Friston KJ, Frith C, Dolan R, Price CJ, Zeki S, Ashburner J, Penny WD (eds) Human brain function. 2nd edn. Academic, New York, pp 695-706

26. Beckmann CF, Smith SM (2004) Probabilistic independent component analysis for functional magnetic resonance imaging. IEEE Trans Med Imaging 23:137-152

27. Friston KJ, Glaser DE, Mechelli A, Turner R, Price CJ (2003) Hemodynamic modeling. In: Frackowiak RSJ, Friston KJ, Frith C et al (eds) Human brain function. Academic, New York, pp 823-842

28. Rosenthal JM, Amiel SA, Yaguez L et al (2001) The effect of acute hypoglycemia on brain function and activation: a functional magnetic resonance imaging study. Diabetes 50:1618-1626
29. Duvernoy HM (1991) The human brain atlas. Springer, Wien

30. Poline JB, Worsley KJ, Evans AC, Friston KJ (1997) Combining spatial extent and peak intensity to test for activations in functional imaging. Neuroimage 5:83-96

31. Gruetter R, Ugurbil K, Seaquist ER (2000) Effect of acute hyperglycemia on visual cortical activation as measured by functional MRI. J Neurosci Res 62:279-285

32. Kringelbach ML, de Araujo IET, Rolls ET (2004) Taste-related activity in the human dorsolateral prefrontal cortex. Neuroimage 21:781-788

33. Rolls ET (2001) The rules of formation of the olfactory representations found in the orbitofrontal cortex olfactory areas in primates. Chem Senses 26:595-604

34. Gordon CM, Dougherty DD, Rauch SL et al (2000) Neuroanatomy of human appetitive function: a positron emission tomography investigation. Int J Eat Disord 27:163-171

35. Uher R, Treasure J, Heining M, Brammer MJ, Campbell IC (2006) Cerebral processing of food-related stimuli: effects of fasting and gender. Behav Brain Res 169:111-119

36. de Araujo IET, Rolls ET, Kringelbach ML, McGlone F, Phillips N (2003) Taste-olfactory convergence, and the representation of the pleasantness of flavour, in the human brain. Eur $\mathrm{J}$ Neurosci 18:2059-2068

37. Rolls ET, Baylis LL (1994) Gustatory, olfactory, and visual convergence within the primate orbitofrontal cortex. J Neurosci 14:5437-5452

38. Critchley HD, Rolls ET (1996) Hunger and satiety modify the responses of olfactory and visual neurons in the primate orbitofrontal cortex. J Neurophysiol 75:1673-1686

39. Rolls ET, Sienkiewicz ZJ, Yaxley S (1989) Hunger modulates the responses to gustatory stimuli of single neurons in the caudolateral orbitofrontal cortex of the macaque monkey. Eur J Neurosci 1:53-60

40. Rolls ET, Yaxley S, Sienkiewicz ZJ (1990) Gustatory responses of single neurons in the caudolateral orbitofrontal cortex of the macaque monkey. J Neurophysiol 64:1055-1066

41. Scott T, Yan J, Rolls E (1995) Brain mechanisms of satiety and taste in macaques. Neurobiology (Bp) 3:281-292

42. Small DM, Gregory MD, Mak YE, Gitelman D, Mesulam MM, Parrish T (2003) Dissociation of neural representation of intensity and affective valuation in human gustation. Neuron 39:701-711

43. Tataranni PA, Gautier JF, Chen KW et al (1999) Neuroanatomical correlates of hunger and satiation in humans using positron emission tomography. PNAS 96:4569-4574

44. Killgore WDS, Yurgelun-Todd DA (2005) Developmental changes in the functional brain responses of adolescents to images of high and low-calorie foods. Dev Psychobiol 47:377-397

45. Rothemund Y, Preuschhof C, Bohner G et al (2007) Differential activation of the dorsal striatum by high-calorie visual food stimuli in obese individuals. Neuroimage 37:410-421

46. Bechara A, Damasio AR, Damasio H, Anderson SW (1994) Insensitivity to future consequences following damage to human prefrontal cortex. Cognition 50:7-15

47. Bechara A, Tranel D, Damasio H (2000) Characterization of the decision-making deficit of patients with ventromedial prefrontal cortex lesions. Brain 123:2189-2202

48. Camille N, Coricelli G, Sallet J, Pradat-Diehl P, Duhamel JR, Sirigu A (2004) The involvement of the orbitofrontal cortex in the experience of regret. Science 304:1167-1170

49. Roberts AC, Reekie Y, Braesicke K (2007) Synergistic and regulatory effects of orbitofrontal cortex on amygdala-dependent appetitive behavior. Ann N Y Acad Sci 1121:297-319

50. Winstanley CA, Theobald DE, Cardinal RN, Robbins TW (2004) Contrasting roles of basolateral amygdala and orbitofrontal cortex in impulsive choice. J Neurosci 24:4718-4722

51. Anderson AW, Heptulla RA, Driesen N et al (2006) Effects of hypoglycemia on human brain activation measured with fMRI. Magn Reson Imaging 24:693-697 